\title{
INVESTIGACIONES
}

\section{Discusión productiva para la comprensión de textos: habilidades y conocimientos específicos en la formación inicial de profesores*}

\author{
Productive Discussions to Support Text Comprehension: \\ Specific Skills and Knowledge in Teachers' Education
}

\author{
Alejandra Meneses A. ${ }^{a}$, Magdalena Müller $A^{b}$., \\ Evelyn Hugo R. ${ }^{c}$, Ángeles García M. ${ }^{d}$ \\ a Pontificia Universidad Católica de Chile. Correo electrónico: amenesel@uc.cl
b Pontificia Universidad Católica de Chile. Correo electrónico: mbmuller@uc.cl
c Pontificia Universidad Católica de Chile. Correo electrónico: eshugo@uc.cl
Pontificia Universidad Católica de Chile. Correo electrónico: angeles.garcia@uc.cl
}

\section{RESUMEN}

La investigación en formación inicial docente ha relevado que los profesores requieren aprender prácticas generativas para una enseñanza efectiva. En este estudio exploratorio de tipo transversal se determinan y caracterizan los desempeños de 125 estudiantes de Pedagogía General Básica en tres momentos de su formación -curso disciplinar (año 1), curso de didáctica (año 2) y práctica (año 3) - en distintas tareas que descomponen la práctica de guiar discusiones para la comprensión lectora: análisis del texto y dificultades para la comprensión, toma de decisiones para facilitar la discusión, identificación de evidencias y errores y observación de una discusión productiva. Este estudio entrega evidencias empíricas para avanzar en la medición de habilidades y conocimientos específicos sobre una práctica generativa crucial para la enseñanza de la lectura; las diferencias significativas encontradas aportan para determinar trayectorias de aprendizaje profesional que integren conocimientos disciplinares y didácticos basados en la práctica.

Palabras clave: práctica generativa, discusión productiva, comprensión de lectura, habilidades y conocimientos específicos, formación inicial de profesores

\section{ABSTRACT}

The teacher education research has revealed that the teachers need to learn high-leverage practices for an effective instruction. This exploratory and cross-sectional study seeks to describe and characterize the 125 Elementary Teaching students' performances in three moments of the initial teacher education disciplinary course (year 1), methods course (year 2) and the field experience (year 3) in different tasks that decompose the practice of facilitating text-based discussion for reading comprehension: text analysis and text difficulties, decision making to facilitate the discussion, identification of evidences and misconceptions, and productive discussion observation. This study contributes with empirical evidences to advance in the assessment of specific skills and knowledge on a crucial high-leverage practice for reading instruction; the significant differences identified help to determine trends of professional learning that integrate disciplinary and pedagogical content knowledge based on practice.

Key words: high-leverage practice, productive discussion, reading comprehension, specialized skills and knowledge, pre-service teacher education

\footnotetext{
* Proyecto desarrollado en el marco del Convenio de Desempeño PUC1201: Innovación en la formación de profesores:
} integración de competencias disciplinarias, pedagógicas y profesionales para la efectividad en las aulas. 


\section{INTRODUCCIÓN}

En la actualidad, la investigación en formación inicial de profesores ha puesto de relieve la necesidad de que los docentes aprendan prácticas profesionales específicas para poder enseñar en contextos diversos desde sus primeros años de formación. Más allá del conocimiento de marcos analíticos y de actividades de reflexión, los profesores requieren de prácticas generativas que les permitan enfrentar la complejidad de una enseñanza que promueva el aprendizaje de todos los estudiantes (Ball y Forzani, 2010; Grossman y McDonald, 2008). Por tanto, las prácticas generativas son un núcleo de acciones esenciales que caracterizan el quehacer de un profesor que fomenta oportunidades de aprendizaje en el aula. Por ejemplo, un profesor que genera aprendizajes se caracteriza por explicar usando modelos, representaciones o ejemplos; guiar discusiones productivas sobre un contenido; facilitar el trabajo en grupos pequeños; seleccionar y utilizar métodos específicos de evaluación; comunicarse con padres o apoderados y analizar la propia práctica con el propósito de mejorarla, entre otras. Estas prácticas surgen de un esfuerzo de sistematización de modos y acciones que caracterizan la profesión docente a partir de evidencias recogidas de la investigación y de la observación de profesores efectivos (Ball y Forzani, 2010).

La práctica generativa de guiar discusiones productivas entre los estudiantes se revela como crucial para la enseñanza con altas expectativas (ambitious teaching); en otras palabras, la discusión productiva es considerada un medio para fomentar el aprendizaje mediante la interacción verbal y el diálogo entre estudiantes con el fin de promover la resolución de problemas complejos y el desarrollo de habilidades cognitivas superiores (Lampert y Graziani, 2009; Thompson, Windschitl y Braaten, 2013). En efecto, la discusión productiva permite extender el razonamiento de los estudiantes mediante el diálogo en la sala de clases.

Dada la relevancia de esta práctica, se vuelve especialmente importante que los profesores novatos la manejen en el contexto de las aulas chilenas. Investigaciones nacionales sobre patrones instruccionales dan cuenta de aulas en que los alumnos tienen una participación verbal restringida, ya que se ven enfrentados mayoritariamente a preguntas cerradas de bajo desafío cognitivo que, en general, están más orientadas a la gestión de aula que al aprendizaje de conceptos y procesos complejos (Facultad de Educación PUC/ MINEDUC, 2011; Preiss, 2009; Radovic y Preiss, 2010). En este contexto, uno de los desafíos que enfrenta la formación inicial de profesores es lograr que prácticas tales como guiar discusiones productivas sean parte del repertorio de enseñanza de los profesores en formación cuando en los contextos escolares auténticos tienen escasas oportunidades de participar en este tipo de interacciones para el aprendizaje. Por lo tanto, ¿cómo conseguir que desarrollen a lo largo de su formación docente, en el espacio universitario, las habilidades y conocimientos específicos para guiar discusiones productivas para el aprendizaje?

Por otra parte, los futuros profesores de básica se enfrentan a otro desafío en el campo de la didáctica específica: ¿cómo desarrollar actividades de comprensión de lectura que permitan que todos los estudiantes comprendan lo que lean? En efecto, en el sistema educativo chileno persiste una gran cantidad de estudiantes de primaria con un bajo nivel de comprensión lectora. Si bien en la formación de profesores en Chile se ha extendido una aproximación didáctica para la enseñanza de la comprensión de lectura basada en las estrategias de antes, durante y después de la lectura, se cuenta con poca evidencia sobre la 
efectividad de esta. En este sentido, resulta relevante explorar la discusión productiva como un tipo de actividad para apoyar la comprensión lectora de los estudiantes. Por lo tanto, poder testear un modelo de discusión basada en un texto como actividad de enseñanza de la lectura no solo permitirá avanzar en la implementación de una práctica generativa para el desarrollo de un profesional de la educación sino también proponer nuevos modos para abordar la compleja tarea de enseñar a leer y leer para aprender.

Este estudio tiene por objetivos determinar y caracterizar el desempeño de estudiantes de Pedagogía General Básica en la práctica generativa sobre la facilitación de una discusión productiva para la comprensión de textos en tres momentos de la formación inicial de estos. Se especifican las habilidades y conocimientos a partir de la descomposición de esta práctica generativa: (1) análisis del texto y dificultades para la comprensión, (2) toma de decisiones para facilitar la discusión, (3) identificación de evidencias y errores y (4) observación de una discusión productiva.

\section{ANTECEDENTES TEÓRICOS Y EMPÍRICOS}

\subsection{LA FORMACIÓN CON FOCO EN PRÁCTICAS GENERATIVAS}

El aprendizaje de prácticas específicas en la formación profesional es un desafío no sólo en la formación de profesores, sino de médicos, abogados, psicólogos, enfermeras, ingenieros, entre otros (Grossman, 2011). Lo que lo hace especialmente difícil de abordar en el caso de la formación de profesores es la carencia de un lenguaje común de la profesión que permita el acceso de los novatos a un cuerpo de conocimiento específico de su campo (Grossman y McDonald, 2008). Por tanto, resulta imperativo avanzar en la definición de prácticas específicas que constituyan un conocimiento relevante y especializado de la profesión y generar las oportunidades para que los estudiantes de pedagogía las puedan aprender a poner en práctica a lo largo de su formación inicial.

Estas prácticas se caracterizan por: (1) estar basadas en la investigación y tener el potencial de impactar en el aprendizaje de los alumnos; (2) ser aplicadas por los principiantes en las salas de clases independiente del currículo y de las metodologías de enseñanza de un contexto particular; (3) poseer una alta frecuencia de uso en las salas de clases y (4) empezar a ser dominadas por un principiante tempranamente (Grossman, Hammerness y McDonald, 2009).

La propuesta de prácticas generativas y de un currículo basado en la práctica permite enfrentar uno de los dilemas de la formación inicial de docentes: la tensión entre teoría y práctica. El situar las prácticas generativas como uno de los ejes de la formación inicial permite alinear los objetivos de los distintos cursos, de tal manera que cada uno de estos desde sus perspectivas diversas contribuya al desarrollo de éstas. Sin embargo, poner el foco en el fomento de estas prácticas supone un desafío a nivel de docencia universitaria, pues se requiere de métodos específicos de aprendizaje adulto para el desarrollo de las prácticas generativas en los distintos cursos del currículo de formación inicial docente. En este sentido, la investigación ha avanzado hacia la definición de elementos centrales de unas pedagogías de la práctica, es decir, métodos que permiten el aprendizaje de habilidades y conocimientos profesionales especializados con distintos niveles de aproximación, pues se asume que el aprendizaje de una práctica generativa es complejo y demandante. 


\subsection{LAS PEDAGOGÍAS DE LA PRÁCTICA EN LA DOCENCIA UNIVERSITARIA}

Para el aprendizaje de estas prácticas específicas, Grossman, Compton et al. (2009) proponen tres conceptos clave que constituyen la base de las pedagogías de la práctica. Por una parte, se refieren a que hay distintas formas de representar las prácticas generativas en la educación profesional. Estas representaciones pueden incluir estudios de casos orales o escritos, videos, libros de textos, evidencias de aprendizaje de estudiantes, entre otros. Por otra parte, postulan que para aprender estas prácticas profesionales específicas es necesario poder descomponerlas en sus partes constitutivas de tal modo que se puedan enseñar de manera intencionada en la formación. Por último, señalan que en la formación profesional se dan distintos tipos de aproximación a la práctica que van desde experiencias menos a más auténticas de aplicación. Entonces, formar profesores con foco en la práctica supone considerar estos elementos en la estructura curricular y en la docencia universitaria, partiendo por la definición de prácticas generativas que sean relevantes de ser enseñadas a los profesores en formación para luego determinar las pedagogías que permitirán el aprendizaje de estas.

\subsection{GUIAR DISCUSIONES PRODUCTIVAS PARA LA COMPRENSIÓN DE TEXTOS}

En la formación de profesores, uno de los dominios clave es el aprendizaje de actividades de enseñanza para el desarrollo de habilidades de comprensión lectora de los estudiantes. Como plantean Kucan y Palincsar (2013), se pueden distinguir por lo menos dos aproximaciones para el aprendizaje de la comprensión lectora. Una aproximación está basada en la enseñanza explícita de estrategias de comprensión lectora con el fin de que los estudiantes las transfieran a diversas situaciones y nuevos contextos de lectura. Esta propuesta didáctica se caracteriza por especificar estrategias para ser utilizadas antes, durante y después de la lectura. Esta aproximación ha tenido una alta difusión en el contexto nacional tanto a nivel de currículo y de recursos escolares como a nivel de sala de clases (Facultad de Educación PUC/MINEDUC, 2011). La otra aproximación de tipo dialógica -con menor difusión- está centrada en la construcción del significado y de la representación coherente de un texto a partir de las distintas ideas que los estudiantes comparten a partir de sus contribuciones verbales en la sala de clases. El objetivo principal de esta propuesta es apoyar a los estudiantes en la formulación y extensión de sus razonamientos mediante la interacción así como construir conocimientos y significados a partir de las ideas formuladas colectivamente a través del diálogo (Kucan y Palincsar, 2013; Murphy, Wilkinson, Soter, Hennessey y Alexander, 2009; Soter et al., 2008). La investigación realizada por McKeon, Beck y Blake (2009) mostró que la aproximación centrada en la discusión sobre el contenido del texto tiene un efecto mayor en la comprensión y en la participación verbal de los estudiantes que la aproximación basada en las estrategias lectoras.

\subsection{DESCOMPOSICIÓN DE LA DISCUSIÓN PRODUCTIVA PARA LA COMPRENSIÓN DE UN TEXTO}

Al considerar los conceptos centrales de las pedagogías de la práctica (Grossman, Compton et al., 2009), con el fin de que los futuros docentes aprendan a guiar satisfactoriamente discusiones productivas para la comprensión de textos, es crucial descomponerla en las 
habilidades y conocimientos específicos que la conforman. En efecto, Kucan, Hapgood y Palincsar (2011) plantean que la discusión productiva para promover la comprensión de lectura en el aula es un espacio instruccional de alta complejidad que requiere de conocimientos especializados. Un desafío adicional es la medición de dichos conocimientos y habilidades que los profesores precisan para guiar una discusión productiva para la comprensión lectora. Kucan et al. (2011) determinaron tres dimensiones clave: (1) análisis del texto y dificultades de la comprensión, (2) identificación de evidencias de comprensión y de errores conceptuales que intervienen en la construcción del significado de lo leído por parte de los estudiantes y (3) formas específicas de responder a las intervenciones de los estudiantes mediante el dominio de movidas pedagógicas de discurso. Para esta investigación, se han considerado y adaptado las dimensiones descritas por Kucan et al. (2011) y, además, se ha incorporado una cuarta dimensión sobre habilidades de observación aplicadas a una situación de discusión productiva a partir de lo propuesto por Müller, Calcagni, Grau, Preiss y Volante (2013). Este tipo de tarea sobre situaciones de enseñanza y aprendizaje permite identificar tanto los focos de observación como los modos de registro y de uso de metalenguaje que utilizan los profesores para referirse a las aulas que analizan.

A continuación se desarrollan las habilidades y conocimientos específicos que se han determinado para la facilitación de la discusión productiva en una actividad de comprensión de textos.

\subsubsection{Análisis del texto y de las dificultades de comprensión para los estudiantes}

Para que los docentes puedan conducir efectivamente una discusión basada en un texto, deben ser capaces, en primer lugar, de analizar el texto seleccionado y dar cuenta del nivel de comprensión que han alcanzado de este. Por lo tanto, el docente debe comprender el texto sobre el cual va a realizar la discusión productiva y al hacerlo pone en funcionamiento sus habilidades como lector. Los lectores que alcanzan niveles profundos de comprensión son capaces no solo de discriminar qué es lo relevante de lo irrelevante, sino también de relacionar la información del texto con su conocimiento previo y de integrar información de distintas secciones de este (Graesser, McNamara y Louwerse, 2003). Por consiguiente, el docente que logra una comprensión profunda del texto podrá guiar más efectivamente a los estudiantes durante la actividad de discusión productiva.

En segundo lugar, los docentes deben ser capaces de identificar las características del texto que va a ser leído en la actividad de discusión. Los textos y la complejidad de estos tienen un efecto importante en la comprensión. De hecho, aquellos con estructura explicativa sobre temáticas de las cuales los lectores disponen de menos conocimientos previos -como los científicos- y con vocabulario académico de uso menos frecuente (McNamara, Ozuru y Floyd, 2011; Snow y Sweet, 2003) son más difíciles de comprender para los estudiantes de educación básica que los textos narrativos. Por lo tanto, los docentes deber ser capaces de identificar las oportunidades así como los obstáculos que presenta el texto escogido para andamiar adecuadamente la comprensión de los estudiantes (Kucan et al., 2011). El docente que facilita una discusión productiva es capaz, entonces, de anticipar las dificultades que los estudiantes experimentarán al intentar comprender el texto escogido para la actividad de enseñanza de la lectura. 


\subsubsection{Toma de decisiones: Foco y movidas pedagógicas para la extensión del discurso y del razonamiento}

El docente para lograr conducir efectivamente la discusión productiva para la comprensión debe ser capaz de generar conexiones entre el foco de la discusión y las ideas clave del texto leído sin perder de vista cómo reconducir la atención de los estudiantes para que logren construir la representación coherente de lo leído (Kucan et al., 2011). Por lo tanto, guiar una discusión implica el análisis y evaluación de las respuestas dadas por los estudiantes durante la discusión. De hecho, las intervenciones de los estudiantes son indicios de los niveles de comprensión que están alcanzando y de las dificultades que están experimentando para lograr la construcción de sentido. Por lo tanto, ser capaz de formular una intervención que focalice la atención de los estudiantes hacia lo central es una tarea de una alta demanda cognitiva.

Por otro lado, guiar la discusión para la comprensión implica que el profesor promueva y facilite la participación verbal de los estudiantes abandonando su rol predominante en el discurso del aula (Kucan et al., 2011). La discusión productiva es un medio que permite que los estudiantes desarrollen procesos de aprendizaje y de alfabetización a través de la interacción en el aula con foco en el desarrollo de las habilidades más complejas de razonamiento, en la extensión de las ideas y en la capacidad para responder a las ideas de los otros a través de la participación verbal (Edwards-Grove, 2014).

Para lograr enriquecer la interacción más allá del patrón inicio, respuesta, evaluación (IRE), el profesor necesita, asimismo, disponer de recursos verbales que le permitan extender el discurso y las ideas de los estudiantes (Beck y McKeon, 2007; Cazden, 2001; Edwards-Grove, 2014; Kucan et al., 2011). Un modo para promover el cambio de los patrones de interacción IRE fuertemente instalados es tomar conciencia de lo que sucede, específicamente, en el tercer turno después de la respuesta dada por los estudiantes e intencionar una intervención que los incite a comprometerse activamente con el desarrollo y extensión de las ideas y de las intervenciones (Beck y McKeon, 2007; Chapin, O’ Connor y Anderson, 2009; Edwards-Grove, 2014).

Las movidas pedagógicas de discurso son estrategias y recursos verbales que cumplen funciones cognoscitivas, interaccionales y socializadoras para construir entornos de aprendizaje dialógicos (Anderson, Chapin y O' Connor, 2011; Edwards-Grove, 2014). Están orientadas, por lo tanto, a incitar a los estudiantes a extender sus razonamientos a través de parafrasear (“Entonces, tú estás diciendo que...”), repetir (“¿Puedes repetir lo que tu compañero ha dicho con tus palabras?”), razonar (“¿Estás de acuerdo? ¿Por qué?”), añadir (“¿Alguien quiere agregar algo más?”), esperar (“Toma tu tiempo”), sostener el razonamiento (“¿Puedes desarrollar más esta idea?”), desafiar el razonamiento (“¿Alguien tiene un punto de vista diferente?") (Beck y McKeon, 2007; Chapin et al., 2009; EdwardsGrove, 2014). Estas movidas de discurso deben ser aprendidas e incorporadas por los docentes para discutir sobre las ideas clave del texto con el fin de desarrollar el pensamiento de los estudiantes y la comprensión profunda y crítica del texto leído.

\subsubsection{Identificación de evidencias de comprensión y errores conceptuales}

Otras habilidades centrales para guiar una discusión productiva para la comprensión del texto dicen relación con la capacidad para detectar y levantar durante la discusión, evidencias del nivel de comprensión que los estudiantes están alcanzando y de las conexiones que estos 
están estableciendo con sus conocimientos previos (Kucan et al., 2011). Asimismo, se debe fomentar la capacidad de los docentes para identificar los modelos iniciales que poseen los estudiantes que interactúan con la información propuesta por el texto y que pueden en ocasiones interferir en la construcción del sentido y del aprendizaje a partir del texto. De este modo, se asume que la comprensión lectora, proceso mental de construcción de significado, necesita ser monitoreada por el docente para poder captar los modos en que los estudiantes están extrayendo e interpretando la información del texto para construir el modelo de situación. Por tanto, las intervenciones verbales de los estudiantes le permiten al profesor darse cuenta de cómo los estudiantes están integrando la información de las distintas partes del texto y cómo están relacionando la información de este con sus conocimientos previos.

\subsubsection{Observación de una discusión productiva}

La habilidad para focalizar una observación en aspectos relevantes de la discusión productiva, el uso del metalenguaje asociado a las movidas pedagógicas y la interpretación de lo observado en base a evidencia (Müller et al., 2013) son también aspectos relevantes de considerar al momento de evaluar el conocimiento sobre la práctica de guiar una discusión productiva. La habilidad de observación supone atender selectivamente a aspectos relevantes de una situación de enseñanza-aprendizaje, así como la capacidad para conectar lo que se observa con conocimiento atingente para describir, interpretar o evaluar lo observado (Santagata y Angelici, 2010). Estas habilidades se han considerado relevantes para la formación docente dado que se ha identificado que al observar interacciones de aula, los profesores expertos atienden más a las evidencias de aprendizaje de los alumnos; en cambio, los profesores novatos seleccionan aspectos superficiales de la enseñanza (Berliner, 1994). Asimismo, una de las actividades de aprendizaje de las prácticas generativas en la lógica de las pedagogías de la práctica dice relación con el análisis de la práctica realizada por otros en situaciones videograbadas.

\section{OBJETIVOS Y PREGUNTAS DE INVESTIGACIÓN}

Este estudio tiene por objetivos determinar y caracterizar el nivel de desempeño obtenido por tres grupos de estudiantes de Pedagogía General Básica en distintos momentos de la formación inicial -curso disciplinar (año 1), curso de didáctica (año 2) y práctica (año 3)sobre cómo facilitar una discusión productiva para la comprensión de textos. Tres preguntas guían este artículo:

(1) ¿Qué nivel de desempeño poseen estudiantes de Pedagogía General Básica que se encuentran en tres momentos distintos de la formación inicial sobre cómo facilitar una discusión productiva para la comprensión de un texto? ¿Qué desempeño alcanzan en las tareas de análisis del texto y dificultades para la comprensión, toma de decisiones para facilitar la discusión, identificación de evidencias de la comprensión y errores conceptuales y observación de una discusión productiva?

(2) ¿Existen diferencias significativas en los desempeños de los estudiantes de Pedagogía General Básica según el momento y curso de la formación inicial -curso disciplinar, curso de didáctica y práctica- en que se encuentran? 
(3) ¿En qué habilidades y conocimientos específicos existen diferencias significativas entre grupos en cada una de las tareas que descomponen la práctica generativa?

\section{MÉTODO}

En este estudio exploratorio de tipo transversal, se determina y caracteriza el desempeño obtenido por estudiantes de Pedagogía General Básica respecto a cómo facilitar una discusión productiva para la comprensión de textos a partir de la descomposición de una práctica generativa clave para el ejercicio profesional.

\subsection{PARTICIPANTES}

La muestra está conformada por 125 estudiantes de Pedagogía en Educación General Básica distribuidos en tres cursos: curso disciplinar del área de lenguaje correspondiente al primer año de carrera (41 estudiantes), curso de didáctica de la lectura del segundo año (53 estudiantes) y, finalmente, la segunda práctica correspondiente al tercer año de carrera (31 estudiantes). Los estudiantes corresponden a tres cohortes distintas del Programa de Pedagogía en Educación Básica en una universidad tradicional ubicada en Santiago de Chile. Los datos fueron recogidos durante el primer semestre del año académico.

El contexto más amplio en que se inserta este estudio es un plan de mejoramiento institucional para aumentar la calidad de la formación inicial docente. La innovación propuesta tiene como objetivo central el rediseño del núcleo formativo con el fin de fomentar las oportunidades de aprendizaje de conocimiento disciplinar y pedagógico disciplinar a partir de un currículo basado en la práctica. El modelo de cambio propone una intervención según tipos de cursos en el que se desarrollan las prácticas generativas a partir de una progresión que considera las diferentes pedagogías de la práctica. Se proponen cursos disciplinares, didácticos y prácticos. En este estudio, se recogen datos en cursos que corresponden precisamente a cada uno de los tres tipos antes descritos.

\subsection{TAREAS}

Cuatro tareas se aplicaron a cada participante; estas se diseñaron para determinar las habilidades y conocimientos especializados para facilitar una discusión productiva para la comprensión de textos. Tres de las tareas fueron traducidas y adaptadas al español -tanto funcional como culturalmente- del instrumento Comprehension and Learning from Text Survey (CoLTS) (Kucan et al., 2011). El texto expositivo escogido para las tres primeras tareas forma parte de un texto escolar de Ciencias destinado a estudiantes de quinto año de enseñanza básica y presenta información visual y verbal sobre el ciclo de vida de las plantas con flores. La cuarta tarea está adaptada de Müller et al. (2013) que consiste en la observación de un video de una discusión realizada por una docente en formación en el contexto de una clase de comprensión de lectura.

Tarea 1: Análisis del texto y dificultades de comprensión. Esta tarea está compuesta por dos preguntas. En la primera pregunta se busca determinar el nivel de comprensión 
alcanzado por los sujetos sobre el texto leído. Para ello deben resumir las ideas principales del texto. Se evalúa la habilidad para (1) identificar ideas principales, (2) identificar ideas secundarias y (3) estructurar un resumen. La segunda pregunta de esta tarea determina cuán capaces son los sujetos para identificar qué aspectos del texto ya sea de (1) contenido, (2) organización y (3) vocabulario pueden resultar difíciles para la comprensión del texto leído. Cada dimensión se puntúa entre 0 y 2, por lo que el puntaje máximo de la tarea es 12 puntos.

Tarea 2: Toma de decisiones para facilitar la discusión. En esta tarea se sitúa al sujeto en escenarios durante una actividad de comprensión de lectura en el aula. Estos son construidos siguiendo dos principios clave: el primero, la segmentación del texto propuesto y el segundo, la formulación de intervenciones por parte de estudiantes que participan de la actividad. Por ejemplo, después de leer la primera parte del texto hasta "la planta de tomate, como toda planta que nace de una semilla, tiene tres etapas en su ciclo de vida", le pides a un estudiante que haga una síntesis de lo comprendido. El estudiante dice: "La vida de un tomate". Mediante esta tarea se determina el nivel de desempeño de los sujetos para (1) estimar y responder a los intentos de los estudiantes para la construcción de significado, es decir, evaluar y explicar qué harían ellos como docentes ante comentarios y respuestas de los estudiantes a preguntas sobre la comprensión de aspectos centrales del texto; y (2) justificar la decisión anterior. Esta tarea está conformada por cuatro preguntas; dos preguntas apuntan a la comprensión de ideas clave del texto y dos, al monitoreo de la comprensión. En esta tarea se miden las habilidades y conocimientos para (1) generar conexión con el foco de la discusión, es decir, se evalúa la capacidad para dirigir la atención de los estudiantes hacia lo central del texto (0-5 puntos), (2) facilitar la participación de los estudiantes mediante la generación de una discusión productiva (0-1 punto), (3) usar movidas pedagógicas específicas (0-1 punto), (4) justificar la intervención propuesta mediante una estrategia para andamiar la comprensión a través del razonamiento y la participación (0-4 puntos) y (5) explicitar el uso de movidas pedagógicas específicas en la justificación (0-1 punto). El puntaje máximo para esta tarea es 48 puntos.

Tarea 3: Identificación de evidencias de la comprensión y errores conceptuales. En esta tarea se determina la capacidad de los sujetos para identificar evidencias sobre la comprensión de lo leído por parte de los estudiantes así como identificar errores conceptuales que interfieran en el aprendizaje a partir del texto. Por ejemplo, después de leer el apartado 2 "Plántula", un estudiante comenta: "El otro día escuché que hay un brote de sarampión”. ¿Qué te indica este comentario con respecto a la comprensión de este estudiante sobre el texto? Esta tarea está conformada por dos preguntas y se puntúa entre 0 y 6 puntos.

Tarea 4: Observación de una discusión productiva. En esta tarea se le pide a los sujetos que describan lo que observan en un video en que un profesor está enseñando estrategias de comprensión de lectura mediante una discusión productiva. Se determina el nivel de desempeño para referirse explícitamente a la discusión productiva como un mecanismo de aprendizaje, mención de una movida pedagógica y ausencia de valoración y de juicio en la respuesta. Esta tarea se puntúa entre 0 y 6 puntos. 


\subsection{PROCEDIMIENTO}

Las tareas se aplicaron mediante un cuestionario on-line de respuesta abierta al cual los estudiantes contestaron voluntariamente durante el primer semestre del año académico. Todos los consentimientos éticos fueron recolectados. La duración aproximada de respuesta del cuestionario fue entre 30 y 45 minutos. Se les preguntó a un grupo de estudiantes sobre su percepción al contestar este instrumento entre las cuales se destaca el desafío de enfrentarse a situaciones de aula y proponer soluciones, el anticipar cómo responderán estudiantes en un contexto de discusión, la cercanía con el ejercicio profesional, entre otras.

Las respuestas fueron codificadas por sujetos capacitados para ello y el $20 \%$ de las respuestas escogidas en forma aleatoria fue doblemente codificado. Los índices Kappa de Cohen obtenidos son considerados muy buenos para cada una de las tareas: tarea $1=.89$, tarea $2=.86$, tarea $3=.85$ y tarea $4=.83$ (Bakerman y Gottman, 1997).

\subsection{PLAN DE ANÁLISIS}

Para responder la primera pregunta de investigación se realizaron estadísticos descriptivos con el fin de dar cuenta de la variabilidad de desempeños obtenidos por los estudiantes en las distintas tareas y según el momento de la formación inicial en que se encuentran los estudiantes de cada cohorte. Para esto se usó el porcentaje de logro por tarea, para poder comparar el desempeño entre estas, ya que poseen puntajes distintos. Una vez verificada la distribución normal de los datos mediante el test de Shapiro-Wilk, se realizó un ANOVA y un test de Bonferroni para comparar el desempeño del puntaje bruto promedio por curso en cada tarea con el fin de contestar a la segunda pregunta de investigación. Finalmente, para observar la existencia de diferencias significativas en habilidades y contenidos específicos por pregunta se realizó una prueba no paramétrica -test chi cuadrado de Pearson- que permite comparar por pregunta si existe independencia o no del desempeño con el curso en que se encuentran los estudiantes.

\section{RESULTADOS}

Esta investigación buscaba determinar y caracterizar el desempeño obtenido por estudiantes de Pedagogía General Básica en tareas que descomponen la práctica de guiar discusiones productivas para la comprensión de textos. Los resultados se organizan para contestar las tres preguntas de investigación que guían este artículo.

\subsection{DESEMPEÑOS PARA GUIAR UNA DISCUSIÓN PRODUCTIVA DURANTE LA COMPRENSIÓN DE TEXTOS}

La primera pregunta apunta a determinar el desempeño obtenido por estudiantes de Pedagogía General Básica en tres momentos de la formación inicial en las habilidades y conocimientos sobre análisis del texto y dificultades para la comprensión, toma de decisiones para facilitar la discusión, identificación de evidencias y errores conceptuales y observación de una discusión productiva. En la tabla 1 se observan los desempeños obtenidos por los estudiantes en las cuatro tareas según el momento de la formación inicial 
en que se encuentran. Con el fin de lograr la comparación entre tareas, se presentan los resultados en porcentaje de logro.

Tabla 1: Desempeño promedio, mínimo y máximo en tareas de descomposición de la discusión productiva por tipo de curso expresados en porcentajes de logro

\begin{tabular}{|c|c|c|c|c|c|c|c|c|c|c|c|c|c|}
\hline \multirow[b]{2}{*}{ Curso } & \multirow[b]{2}{*}{$n$} & \multicolumn{3}{|c|}{$\begin{array}{c}\mathbf{T 1} \\
\text { Análisis texto }\end{array}$} & \multicolumn{3}{|c|}{$\begin{array}{c}\text { T2 } \\
\text { Toma de decisiones }\end{array}$} & \multicolumn{3}{|c|}{$\begin{array}{c}\text { T3 } \\
\text { Evidencias y errores }\end{array}$} & \multicolumn{3}{|c|}{$\begin{array}{c}\text { T4 } \\
\text { Observación }\end{array}$} \\
\hline & & & Min & $\operatorname{Max}$ & & Min & Max & & Min & $\operatorname{Max}$ & & Min & Max \\
\hline $\begin{array}{l}\text { Disciplinar } \\
\text { (1er año) }\end{array}$ & 41 & $60,9 \%$ & 16,7 & 91,7 & $36,7 \%$ & 16,7 & 62,5 & $39,8 \%$ & 0,0 & 83,3 & $26,0 \%$ & 0,00 & 66,67 \\
\hline $\begin{array}{l}\text { Didáctica } \\
\text { (2do año) }\end{array}$ & 53 & $70,4 \%$ & 16,7 & 100 & $45,2 \%$ & 14,6 & 64,6 & $47,8 \%$ & 16,7 & 100 & $34,9 \%$ & 0,00 & 83,33 \\
\hline $\begin{array}{l}\text { Práctica } \\
\text { (3er año) }\end{array}$ & 31 & $64,2 \%$ & 33,3 & 100 & $47,4 \%$ & 25,0 & 66,7 & $43,5 \%$ & 0,0 & 100 & $59,7 \%$ & 16,67 & 100 \\
\hline
\end{tabular}

Como se observa en la tabla 1, las tareas propuestas captan suficiente variabilidad en los desempeños de los estudiantes no solo a través de los cursos sino también al interior de cada uno. Los estudiantes obtuvieron el desempeño más alto en la tarea 1 sobre análisis de texto y dificultades para la comprensión, indistintamente del momento de la formación en que se encuentran. Esto quiere decir que gran parte de los estudiantes logra alcanzar al menos la comprensión de las ideas esenciales del texto propuesto. En cambio, el desempeño obtenido en las otras tres tareas varía según el curso en que se encuentran los profesores en formación.

Los estudiantes de primer año del curso disciplinar obtienen un desempeño promedio más bajo del $50 \%$ en las tres tareas. En la tarea 3 sobre identificación de evidencias y errores conceptuales obtienen un desempeño levemente mayor $(=39,8 \%)$ que en la tarea 2 sobre toma de decisiones para guiar la discusión productiva $(=36,7 \%)$. En la tarea 4 de observación de una discusión productiva obtienen el desempeño más bajo (=26\%). Estos resultados dan cuenta de que las tareas miden habilidades y conocimientos profesionales especializados que los estudiantes de primer año todavía no han tenido oportunidades de desarrollar.

En cuanto a los estudiantes de segundo año que cursan didáctica, se observa un patrón similar al obtenido por los estudiantes de primer año; sin embargo, con un desempeño promedio mayor. Es decir, los estudiantes del curso de didáctica obtienen un desempeño levemente mayor en la tarea 3 de evidencias y errores $(=47,8 \%)$ que en la de toma de decisiones para guiar la discusión $(=45,2 \%)$, mientras que la tarea con menor desempeño es la de observación de una discusión productiva $(=34,9 \%)$.

Por último, los estudiantes que cursan la práctica en el tercer año, en cambio, muestran un desempeño mayor en sus habilidades de observación de una discusión productiva $(=59,7 \%)$ seguido por el desempeño en la tarea sobre toma de decisiones para guiar la discusión $(=47,4 \%)$ e identificación de evidencias y errores $(=43,5 \%)$.

En el Gráfico 1 se pueden observar y comparar los desempeños obtenidos por los estudiantes en las cuatro tareas según momento de la formación inicial en que se encuentran. 
Figura 1: Desempeños de estudiantes de Pedagogía General Básica en tareas de descomposición de la discusión productiva según curso

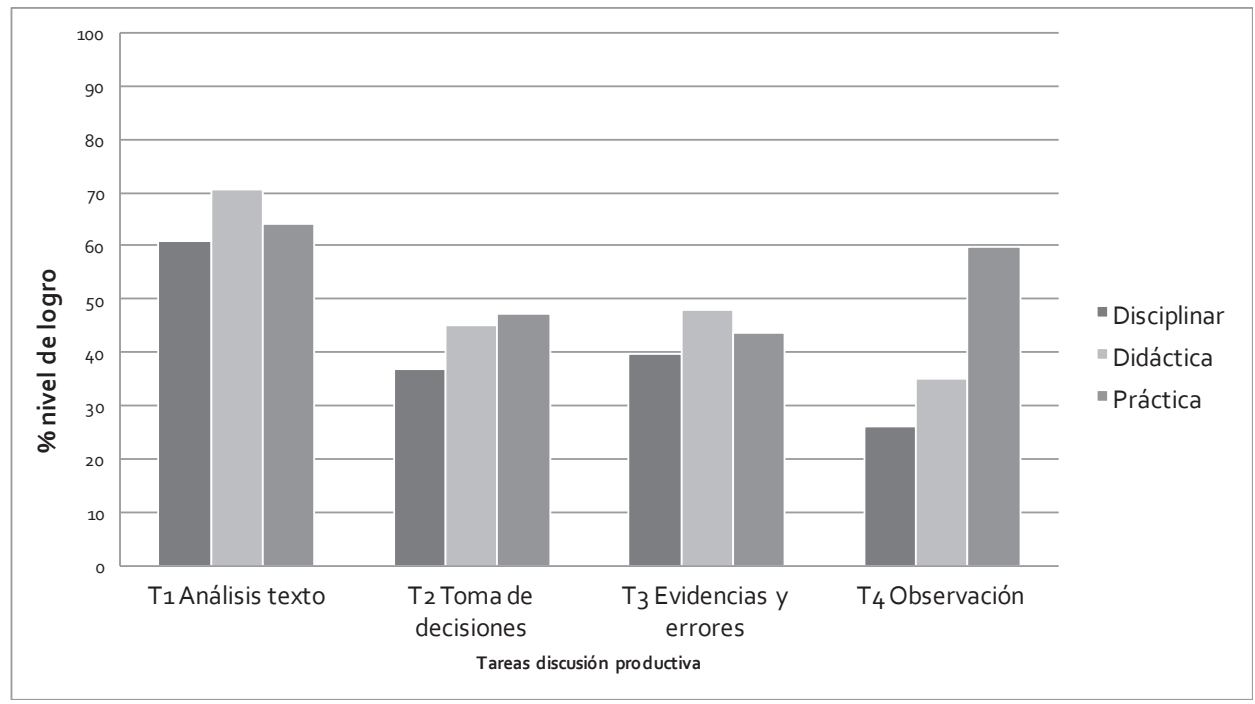

En la figura 1 se observa una tendencia de aprendizaje más definida según momento de la formación inicial en la tarea 2 de toma de decisiones para guiar la discusión productiva y en la tarea 4 sobre habilidades de observación de una discusión productiva. En cambio, en la tarea 1 de análisis de texto y tarea 3 sobre evidencias y errores, los estudiantes de segundo año muestran un desempeño levemente superior. Finalmente, se observa que si bien hay variabilidad en los desempeños, existe todavía una importante zona de aprendizaje de estas habilidades y conocimientos específicos, pues la mayoría de estudiantes aún no alcanza el 50\% de logro.

\subsection{COMPARACIÓN DE DESEMPEÑOS ENTRE CURSOS POR TAREA}

La segunda pregunta de esta investigación busca determinar la existencia de diferencias significativas en los desempeños de los estudiantes de Pedagogía General Básica en las tareas que descomponen la práctica generativa en estudio según el momento de la formación inicial en que se encuentran. Una vez comprobada la distribución normal de los datos mediante el test de Shapiro-Wilk, para la comparación de medias de los puntajes brutos obtenidos en cada tarea por curso, se realizó un análisis de la varianza (ANOVA) de un factor al $95 \%$ de confianza. A partir de este análisis se rechaza la hipótesis de igualdad de las medias de los puntajes por curso, valor- $\mathrm{p}=0,04<0,05$; observándose diferencias significativas en al menos un puntaje promedio respecto a los otros. Las diferencias significativas entre los grupos se observan en la tarea 1 de análisis de texto $[F(2,122)=3,296, \mathrm{p}=.040]$, en la tarea 2 de toma decisiones para guiar la discusión productiva $[F(2,122)=12,076, \mathrm{p}=.000]$ y en la tarea 4 de observación de una discusión productiva $[F(2,122)=19,326, \mathrm{p}=.000]$. No se encontraron diferencias significativas entre cursos en la tarea 3 de evidencias y errores $[F(2,122)=1,568, \mathrm{p}=.213]$. Para constatar entre qué grupos se observan las diferencias, se realizó un análisis de Bonferroni, cuyos resultados se reportan en la tabla 2. 
Tabla 2: Comparación de puntajes promedio entre cursos en cada tarea

\begin{tabular}{|c|c|c|c|c|c|c|c|c|}
\hline \multirow[b]{2}{*}{$\begin{array}{l}\text { Comparación } \\
\text { entre cursos }\end{array}$} & \multicolumn{2}{|c|}{$\begin{array}{c}\mathbf{T 1} \\
\text { Análisis texto }\end{array}$} & \multicolumn{2}{|c|}{$\begin{array}{c}\mathbf{T 2} \\
\text { Toma de decisiones }\end{array}$} & \multicolumn{2}{|c|}{$\begin{array}{c}\text { T3 } \\
\text { Evidencias y errores }\end{array}$} & \multicolumn{2}{|c|}{$\begin{array}{c}\text { T4 } \\
\text { Observación }\end{array}$} \\
\hline & $\begin{array}{l}\text { Diferencia } \\
\text { de medias }\end{array}$ & Valor-p & $\begin{array}{l}\text { Diferencia } \\
\text { de medias }\end{array}$ & Valor-p & $\begin{array}{l}\text { Diferencia } \\
\text { de medias }\end{array}$ & Valor-p & $\begin{array}{l}\text { Diferencia } \\
\text { de medias }\end{array}$ & Valor-p \\
\hline $\begin{array}{l}\text { Disciplinar- } \\
\text { Didáctica }\end{array}$ & $-1,136$ & $.04^{*}$ & $-4,064$ & $.000 * * *$ & $-0,478$ & .242 & $-0,533$ & .207 \\
\hline $\begin{array}{l}\text { Didáctica- } \\
\text { Práctica }\end{array}$ & 0,743 & .401 & $-1,044$ & 1 & 0,255 & 1 & $-1,486$ & $.000 * * *$ \\
\hline $\begin{array}{l}\text { Práctica- } \\
\text { Disciplinar }\end{array}$ & 0,393 & 1 & 5,108 & $.000 * * *$ & 0,223 & 1 & 2,02 & $.000 * * *$ \\
\hline
\end{tabular}

Este análisis más específico permite indagar en forma particular entre qué cursos se observan diferencias significativas en cada tarea. En relación a la tarea 1 de análisis de texto se encuentran diferencias significativas entre el curso disciplinar y el de didáctica y, presentan mejor desempeño los estudiantes del curso de didáctica que los del disciplinar. En la tarea 2 de toma de decisiones, las diferencias significativas se observaron entre el curso disciplinar y el de didáctica, y obtuvieron mejores resultados los estudiantes del curso de didáctica que los del disciplinar; y entre el curso disciplinar y la práctica, con mejor desempeño por parte de los estudiantes de la práctica. En la tabla 3, se muestran respuestas de estudiantes que evidencian las diferencias entre cursos.

Tabla 3: Respuestas por curso en pregunta de la Tarea 2

\begin{tabular}{|c|c|c|c|}
\hline Pregunta & $\begin{array}{l}\text { Después de leer el apartado } \\
\text { cómo se reproduce una plan } \\
\text { ¿Cómo responderías a esta } \mathrm{r}\end{array}$ & $\begin{array}{l}\text { "1. Semilla", usted le pide a un e } \\
\text { ta. El estudiante responde: "por las } \\
\text { espuesta del estudiante? }\end{array}$ & $\begin{array}{l}\text { studiante voluntario que explique } \\
\text { semillas". }\end{array}$ \\
\hline \multirow[b]{2}{*}{ Respuesta } & $\begin{array}{l}\text { Curso disciplinar } \\
\text { (Primer año) }\end{array}$ & $\begin{array}{l}\text { Curso didáctica } \\
\text { (Segundo año) }\end{array}$ & $\begin{array}{l}\text { Curso práctica } \\
\text { (Tercer año) }\end{array}$ \\
\hline & $\begin{array}{l}\text { Que está muy bien su } \\
\text { respuesta. }\end{array}$ & $\begin{array}{l}\text { Respondería diciéndole que está } \\
\text { bien, pero que sea más claro, } \\
\text { guiándolo un poco. Por ejemplo } \\
\text { preguntándole: “ ¿Dónde se } \\
\text { encuentran estas semillas? } \\
\text { ¿Qué originan las semillas?” }\end{array}$ & $\begin{array}{l}\text { "Muy bien, la planta de tomate } \\
\text { se reproduce por semillas que se } \\
\text { encuentran dentro del fruto } \\
\text { pero, ¿cómo ocurre este } \\
\text { proceso?" }\end{array}$ \\
\hline Análisis & $\begin{array}{l}\text {-No hace mención a las } \\
\text { ideas del texto. } \\
\text {-En la intervención, evalúa } \\
\text { cerrando la interacción. } \\
\text {-No usa explícitamente } \\
\text { ninguna movida } \\
\text { pedagógica de discurso. }\end{array}$ & $\begin{array}{l}\text {-Focaliza la atención en } \\
\text { localizar las semillas dentro de } \\
\text { la planta adulta. } \\
\text {-En la intervención, pregunta } \\
\text { extendiendo la interacción. } \\
\text {-No usa explícitamente ninguna } \\
\text { movida pedagógica de discurso. }\end{array}$ & $\begin{array}{l}\text {-Focaliza la atención en explicar } \\
\text { cómo o por qué se reproduce la } \\
\text { planta. } \\
\text {-En la intervención, pregunta } \\
\text { extendiendo la interacción. } \\
\text {-No usa explícitamente ninguna } \\
\text { movida pedagógica de discurso. }\end{array}$ \\
\hline
\end{tabular}


Con respecto a la tarea 3 de evidencias y errores no se observaron diferencias significativas entre los cursos. En la tarea 4 de observación, se encontraron diferencias significativas entre el curso disciplinar y la práctica así como entre el curso de didáctica y la práctica, con mejor desempeño por parte de los estudiantes de la práctica.

\subsection{DIFERENCIAS EN HABILIDADES Y CONOCIMIENTOS ESPECÍFICOS PARA GUIAR LA DISCUSIÓN PRODUCTIVA}

La tercera pregunta de esta investigación busca determinar específicamente en qué habilidades y conocimientos para guiar una discusión productiva existen diferencias significativas entre grupos por tarea. Se realizó un test chi cuadrado de Pearson $\left(\chi^{2}\right)$ para observar si existe dependencia entre la habilidad y el conocimiento específico medido a nivel de la pregunta y el curso en el cual se encuentra el estudiante. En la tabla 4 se reportan las diferencias para cada una de las habilidades y conocimientos involucrados en el dominio de esta práctica generativa.

Tabla 4: Diferencias significativas entre grupos por habilidades y conocimientos específicos por tarea

\begin{tabular}{llcl}
\hline \multicolumn{1}{c}{ Tarea } & Habilidad y conocimiento a nivel de pregunta & $\chi^{2}$ & p-valor \\
\hline \multirow{4}{*}{ Análisis } & P1. Identificar ideas principales & 13,36 & $.010^{* *}$ \\
texto & P1. Identificar ideas secundarias & 5,6 & .469 \\
& P1. Estructurar resumen & 15,04 & $.005^{* *}$ \\
& P2. Explicitar dificultades sobre el contenido del texto & 0,91 & .923 \\
& P2. Explicitar dificultades sobre la organización del texto & 4,98 & .289 \\
& P2. Explicitar dificultades sobre el vocabulario del texto & 5,13 & .274 \\
\hline \multirow{6}{*}{ P3. Generar conexión con foco de la discusión } & 26,03 & $.011^{*}$ \\
& P3. Facilitar la participación de los estudiantes & 8,81 & $.012^{*}$ \\
& P3. Usar movidas pedagógicas específicas & 1,74 & .419 \\
& P3. Justificar intervención propuesta & 11,84 & .298 \\
Toma de & P3. Explicitar uso movida pedagógica & 0,75 & .686 \\
decisiones & 25,87 & $.011^{*}$ \\
& P4. Generar conexión con foco de la discusión & 20,25 & $.000^{* * *}$ \\
& P4. Eacilitar la participación de los estudiantes & 6,25 & $.044 *$ \\
& P5. Generar conexión con foco de la discusión & 20,82 & $.008^{* *}$ \\
& P4. Eacilitar la participación de los estudiantes & 3,05 & .217 \\
& P5. Usartificar intervención propuesta & 15,02 & $.020^{*}$ \\
& P5. Usaridas pedagógicas específicas & 4,03 & .133 \\
& P5. Justificar intervención propuesta & 0,71 & .698 \\
& P5. Explicitar uso movida pedagógica & 12,39 & .134 \\
& P6. Generar conexión con foco de la discusión & 11,75 & $.003^{* *}$ \\
& P6. Facilitar la participación de los estudiantes & 13,62 & .191 \\
& P6. Usar movidas pedagógicas específicas & 32,12 & $.000^{* * *}$ \\
& P6. Justificar intervención propuesta & 18,74 & $.000^{* * *}$ \\
& P6. Explicitar uso movida pedagógica & 25,955 & $.004^{* *}$ \\
& & 8,86 & $.012^{*}$ \\
\hline
\end{tabular}




\begin{tabular}{llcl}
\hline \multirow{2}{*}{3} & P7. Identificar evidencias de la comprensión de un estudiante & 26,67 & $.001^{* *}$ \\
Evidencias & P7. Identificar error del estudiante & 5,96 & .202 \\
y errores & P8. Identificar evidencias de la comprensión de dos estudiantes & 7,07 & .132 \\
& P8. Identificar el error del estudiante 1 & 2,59 & .628 \\
& P8. Identificar el error del estudiante 2 & 2,95 & .565 \\
\hline \multirow{2}{*}{4} & P9. Describir elementos de la discusión productiva & 30,09 & $.000^{* * *}$ \\
\multirow{2}{*}{ Observación } & P9. Explicitar uso de movida pedagógica & 34,75 & $.000^{* * *}$ \\
& P9. Describir sin juicio de valor & 15,46 & $.004^{* *}$ \\
\hline
\end{tabular}

Nota. $* \mathrm{p} \geq .05 ; * * \mathrm{p} \geq .01, * * * \mathrm{p} \geq .000 . \mathrm{P}=$ número de pregunta

En la tabla 4 se observan que existen diferencias significativas en 18 de las 34 habilidades y conocimientos específicos para guiar la discusión productiva evaluados. En otras palabras, se rechaza la hipótesis de que la habilidad y el conocimiento medidos y el momento de la formación en que se encuentra el estudiante son independientes. Por lo tanto, se puede afirmar que dichos conocimientos y habilidades tienen un comportamiento diferente dependiendo el curso en el que el estudiante se encuentra.

En la tarea 1 de análisis del texto y dificultades de comprensión, las diferencias significativas se encontraron, específicamente, en la habilidad para identificar ideas principales y estructurar un resumen. En la tarea 2 de toma de decisiones para facilitar la discusión, se encontraron diferencias significativas en las habilidades de generar conexión con el foco de la discusión que determina la capacidad para redirigir la atención de los estudiantes hacia las ideas principales del texto en tres de las cuatro preguntas. El mismo patrón se encontró en facilitar la participación de los estudiantes utilizando para ello una pregunta que permite la intervención verbal de estos. En relación al uso de movidas pedagógicas específicas para extender las respuestas de los estudiantes así como en la explicitación de estas en la justificación dada, se encontró que hubo diferencias significativas en dos de las cuatro preguntas. El mismo patrón se encontró en la especificidad de la justificación dada a la respuesta propuesta. En la tarea 3 de identificación de evidencias y errores conceptuales solo se encontró diferencias significativas en la habilidad para levantar evidencias de comprensión lectora vinculada al conocimiento previo y al vocabulario cotidiano. Por último, en la tarea 4 de observación de una discusión productiva se encontraron diferencias significativas en las tres habilidades medidas: descripción de elementos de una discusión productiva, explicitación de movidas pedagógicas y descripción sin juicio de valor. En la tabla 5, se muestran respuestas a la pregunta de observación que evidencian las diferencias a nivel de habilidades y conocimientos especializados. 
Tabla 5: Respuestas por curso en pregunta de la Tarea 4

\begin{tabular}{|c|c|c|c|}
\hline Pregunta & ¿Qué ves en este video? & & \\
\hline \multirow[t]{2}{*}{ Respuesta } & $\begin{array}{l}\text { Curso disciplinar } \\
\text { (Primer año) }\end{array}$ & $\begin{array}{l}\text { Curso didáctica } \\
\text { (Segundo año) }\end{array}$ & $\begin{array}{l}\text { Curso práctica } \\
\text { (Tercer año) }\end{array}$ \\
\hline & $\begin{array}{l}\text { Veo que la profesora solo } \\
\text { se mueve por un sector de } \\
\text { la sala de manera } \\
\text { hiperquinética ayudando } \\
\text { poco a la concentración } \\
\text { de los niños. }\end{array}$ & $\begin{array}{l}\text { La profesora incentiva el } \\
\text { uso de un ordenador } \\
\text { gráfico, hace participar a } \\
\text { los estudiantes } \\
\text { constantemente y da } \\
\text { feedback en caso de error. }\end{array}$ & $\begin{array}{l}\text { Se utilizan estrategias para promover la } \\
\text { discusión (movidas pedagógicas). } \\
\text { Mediante preguntas se promueve el } \\
\text { razonamiento de los alumnos y se los } \\
\text { motiva a posicionarse sobre distintas } \\
\text { perspectivas para resolver un problema. } \\
\text { La docente emplea estrategias en los } \\
\text { alumnos para poder razonar (“¿Qué } \\
\text { crees que sería mejor?”), añadir } \\
\text { (“¿Cuál otra razón darías para ocupar } \\
\text { un organizador gráfico?”), tomar } \\
\text { posturas (“¿Estás de acuerdo en hacer } \\
\text { un resumen en otra hoja?”), entre otras. }\end{array}$ \\
\hline Análisis & $\begin{array}{l}\text {-No alude a la discusión } \\
\text { productiva como } \\
\text { mecanismo de } \\
\text { aprendizaje. } \\
\text {-No menciona ninguna } \\
\text { movida pedagógica. } \\
\text {-Incluye valoración. }\end{array}$ & $\begin{array}{l}\text {-Menciona evidencias de } \\
\text { interacción pero no se } \\
\text { explicita que se trate de } \\
\text { una discusión productiva. } \\
\text {-No menciona ninguna } \\
\text { movida pedagógica. } \\
\text {-Describe sin incluir } \\
\text { juicios u opiniones } \\
\text { respecto a esto. }\end{array}$ & $\begin{array}{l}\text {-Menciona explícitamente la discusión } \\
\text { productiva. } \\
\text {-Menciona explícitamente movidas } \\
\text { pedagógicas. } \\
\text {-Describe sin incluir juicios u opiniones } \\
\text { respecto a esto. }\end{array}$ \\
\hline
\end{tabular}

\section{CONCLUSIONES Y DISCUSIONES}

En este artículo se determinan y caracterizan los desempeños obtenidos por estudiantes de Pedagogía General Básica en tres momentos de la formación en distintas tareas que descomponen la práctica generativa de guiar discusiones para la comprensión de textos. Las tareas adaptadas y ajustadas de Kucan et al. (2011) y Müller et al. (2013) para medir las habilidades y conocimientos especializados involucrados en la práctica generativa en estudio logran captar variabilidad de desempeños tanto a través de los cursos como al interior de cada uno, lo que permite disponer de evidencias iniciales para determinar trayectorias de dominio profesional y discriminar entre desempeños. Esta investigación contribuye a mostrar cómo se integran dos dimensiones clave del quehacer profesional docente: aproximación didáctica para la comprensión de lectura más allá de la enseñanza explícita de estrategias de lectura y la discusión productiva como un tipo de interacción de aula que permite extender el razonamiento y el discurso de los estudiantes para el aprendizaje.

En el contexto actual que enfatiza el desarrollo de prácticas generativas específicas en la formación inicial de profesores, este estudio contribuye a abordar el desafío de evaluarlas. En primer lugar, considerando uno de los conceptos clave para el aprendizaje profesional descritos por Grossman, Compton et al. (2009), se ha avanzado en la descomposición de la práctica de guiar una discusión productiva para la comprensión de un texto, en tareas 
específicas susceptibles de ser enseñadas y evaluadas en la formación. Los profesores en formación deberían contar con oportunidades para: 1) analizar un texto y las dificultades de comprensión asociadas a este, 2) tomar decisiones para la facilitación de la discusión, 3 ) identificar evidencias de la comprensión y errores conceptuales y 4) observar e interpretar lo observado de acuerdo a su conocimiento sobre las características de una discusión productiva que promueve la construcción de significado en torno a un texto. El objetivo de esta descomposición es que los estudiantes puedan tener experiencias que les permitan avanzar en el desempeño de los conocimientos y habilidades específicas implicadas en su ejecución. Los resultados de este estudio muestran que las tareas y las mediciones son promisorias para determinar los desempeños en la práctica generativa en estudio.

De las cuatro tareas evaluadas, los estudiantes obtuvieron el mejor desempeño en la tarea 1 de análisis de texto y dificultades para la comprensión. Esto quiere decir que gran parte de los estudiantes logra alcanzar al menos la comprensión de las ideas esenciales del texto propuesto. En las otras tres tareas evaluadas, en cambio, los estudiantes del curso disciplinar del primer semestre de formación presentan un desempeño promedio más bajo que los otros dos grupos evaluados. Esto podría estar dando cuenta de que estas tres tareas estarían midiendo habilidades y conocimientos profesionales especializados que se van desarrollando en el transcurso de la formación. Por otra parte, también se observa que si bien hay variabilidad en los desempeños, existe todavía una importante zona de desarrollo de estas habilidades y conocimientos específicos, pues la mayoría de estudiantes aún no alcanza el 50\% de logro más allá del momento de la formación en que se encuentre.

Al comparar el desempeño de los tres cursos por tareas, se observan diferencias significativas entre los grupos en las tareas 1, 2 y 4 . En cambio, en la tarea 3 que mide conocimientos y habilidades en relación a la identificación de evidencias de la comprensión $y$ de errores conceptuales, no se observaron diferencias significativas entre los grupos a nivel general.

Al hacer un análisis más específico para comparar las habilidades y conocimientos que componen la tarea 3 de evidencias y errores se observa que sólo hay diferencias significativas entre los grupos en la identificación de evidencias de comprensión de los estudiantes. Por otra parte, esto es consistente con lo observado en la tarea 1 de análisis del texto en que a nivel más específico se puede observar que las diferencias significativas se dan en relación a la identificación de ideas principales y la estructuración de un resumen pero no en las habilidades y conocimientos relacionados con explicitar dificultades en relación al contenido, a la organización y al vocabulario del texto. Esto podría indicar la necesidad de generar intencionadamente más oportunidades para el aprendizaje que permita la integración explícita entre el conocimiento disciplinar y pedagógico en la formación docente. De la tarea 2 de toma de decisiones y de la tarea 4 de observación se concluye que existen diferencias significativas, por una parte, en el uso de movidas pedagógicas para redirigir la atención de los estudiantes hacia las ideas clave a través de preguntas que buscan extender la participación de los estudiantes y, por otra parte, la explicitación de un metalenguaje que da cuenta del conocimiento específico sobre la discusión productiva adquirido.

Los resultados de esta investigación son consistentes con los hallazgos de Kucan et al. (2011) con profesores estadounidenses en ejercicio. La mayoría de los profesores de dicho estudio mostraron desempeños bajos en las habilidades para identificar y anticipar recursos del texto vinculados al vocabulario, estructura y contenido que podrían obstaculizar la comprensión de este. Asimismo, encontraron que los profesores poseen un conocimiento 
limitado sobre movidas pedagógicas de discurso para promover la discusión sobre textos o bien mencionan movidas poco eficaces para redirigir la atención de los estudiantes hacia las ideas clave del texto. Por lo tanto, las autoras proponen que los profesores tanto en ejercicio como en formación requieren de oportunidades de aprendizaje que les permitan alcanzar el dominio de esta práctica generativa de alta complejidad. Una de las principales contribuciones del presente estudio es, entonces, determinar diferencias significativas en los desempeños entre grupos a nivel de tarea y a nivel de habilidades y conocimientos específicos que descomponen cada una de las tareas. De este modo, la descomposición de la discusión productiva y la evaluación de la misma permite sentar las bases para un estudio futuro de tipo longitudinal que permita determinar trayectorias de aprendizaje profesional.

En cuanto a las diferencias en las habilidades de observación de los docentes en formación, los resultados obtenidos en este estudio son similares a los de Müller et al. (2013). Los estudiantes avanzan en sus habilidades para focalizar sus observaciones en las oportunidades de aprendizaje en el aula y pasan de descripciones generales a específicas sin uso de evaluaciones.

Este estudio aporta evidencias empíricas para avanzar en la medición de habilidades y conocimientos específicos sobre prácticas generativas tanto de profesores en ejercicio como en formación en la que se integran no solo conocimientos disciplinares sino también conocimientos propios de la didáctica. Sin embargo, quedan desafíos pendientes sobre cómo se relacionan estos desempeños de habilidades y conocimientos específicos medidos en situaciones hipotéticas con el desempeño al ejecutar la discusión productiva en un determinado contexto y más aún cómo dichos desempeños se relacionan con el desarrollo de las habilidades de comprensión lectora de estudiantes del sistema escolar.

\section{AGRADECIMIENTOS}

Las autoras agradecen a los codificadores, a los académicos que abrieron sus aulas, a los estudiantes que participaron de este estudio y a Alejandra Venegas por el apoyo en los análisis estadísticos.

\section{REFERENCIAS BIBLIOGRÁFICAS}

Anderson, N., Chapin, S. y O' Connor, C. (2011). Classroom discussions: Seeing Math discourse in action (multimedia professional learning resource). Sausalito, CA: Math Solutions.

Bakerman, R. y Gottman, J. M. (1997). Assessing observer agreement. En R. Bakerman, y J. M. Gottman. (Eds.), Observing interaction: An introduction to sequential analysis (pp. 56-80). Cambridge: University Press.

Ball, D. L. y Forzani, F. M. (2010). What does it take to make a teacher? Phi Delta Kappan, vol. 92 (2), 8-12. doi: 10.1177/003172171009200203

Beck, I. L. y McKeon, M. G. (2007). How teachers can support productive classroom talk: Move the thinking to the students. En R. Horowitz (Ed.), Talking texts (pp. 207-220). Mahwah, NJ: Lawrence Erlabum.

Berliner, D. C. (1994). Expertise: The wonder of exemplary performances. En M. J. N y C. C. Block (Eds.), Creating powerful thinking in teachers and students: Diverse perspectives (pp. 161-186). Orlando, FL: Harcourt Brace. 
Cazden, C. (2001). Classroom discourse. The language of teaching and learning. Portsmouth, NH: Heinemann.

Chapin, S., O’ Connor, C. y Anderson, N. (2009). Classroom discussions: Using Math talk to help students learn, grades $K-6$. Sausalito, CA: Math Solutions.

Edwards-Grove, C. (2014). Talk moves: A repertoire of practices for productive classroom dialogue. Primary English Teaching Association Australia PETAA Paper, 195, 1-12.

Graesser, A., McNamara, D. y Louwerse, M. (2003). What do readers need to learn in order to process coherence relations in narrative and expository text. In A. Sweet \& S. C. (Eds.), Rethinking reading comprehension (pp. 82-98). New York, NJ: Guilford Publications.

Grossman, P. (2011). Framework for Teaching Practice: A Brief History of an Idea. Teachers College Record, vol. 113 (9), 2836-2843.

Grossman, P., Compton, C., Igra, D., Ronfeldt, M., Shahan, E. y Williamson, P. W. (2009). Teaching Practice: A Cross-Professional Perspective. Teachers College Record, vol. 111 (9), 2055-2100.

Grossman, P., Hammerness, K. y McDonald, M. (2009). Redefining teaching, re-imagining teacher education. Teachers and Teaching, vol. 15 (2), 273-289. doi: 10.1080/13540600902875340

Grossman, P. y McDonald, M. (2008). Back to the future: Directions for research in teaching and teacher education. American Educational Research Journal, vol. 45 (1), 184-205. doi: 10.3102/0002831207312906

Kucan, L., Hapgood, S. y Palincsar, A. S. (2011). Teachers' specialized knowledge for supporting student comprehension in text-based discussions. Elementary School Journal, vol. 112 (1), 6182. doi: $10.1086 / 660689$

Kucan, L. y Palincsar, A. (2013). Comprehension instruction through text-based discussion. Newark, DE: International Reading Association.

Lampert, M. y Graziani, F. (2009). Instructional activities as a tool for teachers' and teacher educators' learning. Elementary School Journal, vol. 109 (5), 491-509. doi: 10.1086/596998

McKeown, M. G., Beck, I. L. y Blake, R. G. K. (2009). Rethinking reading comprehension instruction: A comparison of instruction for strategies and content approaches. Reading Research Quarterly, vol. 44 (3), 218-253. doi: 10.1598/rrq.44.3.1

McNamara, D., Ozuru, Y. y Floyd, R. (2011). Comprehension challenges in the fourth grade: The roles of text cohesion, text genre and readers' prior knowledge. International Electronic Journal of Elementary Education, vol. 4 (1), 229-257.

Facultad de Educación PUC/MINEDUC. (2011). Alfabetización en establecimientos chilenos subvencionados (Informe final). Santiago de Chile: Facultad de Educación, Pontificia Universidad Católica de Chile.

Murphy, P. K., Wilkinson, I. A. G., Soter, A. O., Hennessey, M. N. y Alexander, J. F. (2009). Examining the effects of classroom discussion on students' comprehension of text: A metaanalysis. Journal of Educational Psychology, vol. 101 (3), 740-764. doi: 10.1037/a0015576

Müller, M., Calcagni, E., Grau, V., Preiss, D. D. y Volante, P. (2013). Desarrollo de habilidades de observación en estudiantes de pedagogía: Resultados de una intervención piloto basada en el uso de la Videoteca de Buenas Prácticas Docentes. Estudios pedagógicos vol. 39 (Especial), 85-101. doi: 10.4067/s0718-07052013000300007

Preiss, D. D. (2009). The Chilean instructional pattern for the teaching of language: A video-survey study based on a national program for the assessment of teaching. Learning and Individual Differences, vol. 19 (1), 1-11. doi: 10.1016/j.lindif.2008.08.004

Radovic, D. y Preiss, D. (2010). Patrones de discurso observados en el aula de matemática de segundo ciclo básico en Chile. Psykhe, vol. 19 (2), 66-79. doi: 10.4067/S0718-222820100002000007

Santagata, R. y Angelici, G. (2010). Studying the impact of the lesson analysis framework on preservice teachers' abilities to reflect on videos of classroom Teaching. Journal of Teacher Education, vol. 61 (4), 339-349. doi: 10.1177/0022487110369555

Snow, C. y Sweet, A. (2003). Reading for comprehension. En A. Sweet y C. Snow (Eds.), Rethinking 
Estudios Pedagógicos XLII, N 4: 87-106, 2016

DISCUSIÓN PRODUCTIVA PARA LA COMPRENSIÓN DE TEXTOS: HABILIDADES Y CONOCIMIENTOS ESPECÍFICOS EN LA FORMACIÓN INICIAL DE PROFESORES

reading comprehension (pp. 1-11). New York, NJ: Guilford Press.

Soter, A. O., Wilkinson, I. A., Murphy, P. K., Rudge, L., Reninger, K. y Edwards, M. (2008). What the discourse tells us: Talk and indicators of high-level comprehension. International Journal of Educational Research, vol. 47 (6), 372-391. doi: 10.1016/j.ijer.2009.01.001

Thompson, J., Windschitl, M. y Braaten, M. (2013). Developing a theory of ambitious earlycareer teacher practice. American Educational Research Journal, vol. 50 (3), 574-615. doi: 10.3102/0002831213476334 